1

MS \#JHEPAT-D-09-00085-R1

\title{
Association Analysis of Cytotoxic T-lymphocyte Antigen 4 Gene \\ Polymorphisms with Primary Biliary Cirrhosis in Japanese Patients
}

\author{
Satoru Joshita ${ }^{1}$, Takeji Umemura ${ }^{1}$, Kaname Yoshizawa ${ }^{1}$, Yoshihiko Katsuyama ${ }^{2}$, \\ Eiji Tanaka ${ }^{1}$, Minoru Nakamura ${ }^{3}$, Hiromi Ishibashi ${ }^{3}$, Masao Ota ${ }^{4}$ and the Shinshu PBC \\ Study Group * \\ 1 Department of Medicine, Division of Gastroenterology and Hepatology, Shinshu \\ University School of Medicine, 3-1-1 Asahi, Matsumoto, 390-8621, Japan \\ ${ }^{2}$ Department of Pharmacy, Shinshu University Hospital, 3-1-1 Asahi, Matsumoto, \\ 390-8621, Japan \\ ${ }^{3}$ Clinical Research Center, National Hospital Organization (NHO) Nagasaki Medical \\ Center and Department of Hepatology, Nagasaki University Graduate School of \\ Biomedical Sciences, 2-1001-1 Kubara, Omura, 856-8562, Japan \\ ${ }^{4}$ Department of Legal Medicine, Shinshu University School of Medicine, 3-1-1 Asahi, \\ Matsumoto, 390-8621, Japan
}


Short title: CTLA4 polymorphisms in Japanese PBC patients

\section{Corresponding author:}

Takeji Umemura, MD, PhD, Department of Medicine, Division of Gastroenterology and Hepatology, Shinshu University School of Medicine, 3-1-1 Asahi, Matsumoto, 390-8621, Japan

E-mail: tumemura@shinshu-u.ac.jp

Tel.: +81-263-37-2634

Fax: $+81-263-32-9412$

Electronic word count: 4604

Number of figures and tables: No figures and six tables

List of abbreviations : primary biliary cirrhosis, PBC; anti-mitochondrial antibody, AMA; cytotoxic T-lymphocyte antigen 4, CTLA4; orthotopic liver transplantation, OLT; single 
nucleotide polymorphisms, SNPS; untranslated region, UTR; linkage disequilibrium, LD; Hardy-Weinberg Equilibrium, HWE; Pc, corrected P; odds ratio, OR; confidence interval, Cl; soluble isoform of CTLA4, sCTLA4.

Financial disclosure: The authors declare that they have nothing to disclose regarding funding from industries or conflicts of interest with respect to this manuscript.

* The Shinshu PBC Study Group: Michiharu Komatsu, Naoki Tanaka, Tetsuya Ichijo, Akihiro Matsumoto 


\begin{abstract}
Background/Aims: Primary biliary cirrhosis (PBC) is an organ-specific autoimmune disease of still unidentified genetic etiology that is characterized by chronic inflammation of the liver. Since cytotoxic T-lymphocyte antigen 4 (CTLA4) polymorphisms have recently been linked with $\mathrm{PBC}$ susceptibility in studies on Caucasians, we investigated the genetic association between CTLA4 polymorphisms and PBC in a Japanese population.
\end{abstract}

Methods: Five single nucleotide polymorphisms (SNPs) in the CTLA4 gene (rs733618, rs5742909, rs231775, rs3087243, and rs231725) were genotyped in $\underline{308}$ patients with PBC and $\underline{268}$ healthy controls using a TaqMan assay.

Results: One CTLA4 gene SNP (rs231725) was significantly associated with susceptibility to anti-mitochondrial antibody (AMA)-positive PBC, but clinical significance disappeared after correction for multiple testing. Moreover, CTLA4 gene SNPs did not influence AMA development or disease progression to orthotopic liver transplantation in our Japanese cohort. In haplotype analyses, one haplotype [haplotype 1 (CGGA)] at rs5742909, rs231775, rs3087243, and rs231725, was significantly associated with susceptibility to both AMA-positive PBC and overall PBC.

Conclusions: This study showed that CTLA4 gene polymorphisms had a modest, 
but significant, association with susceptibility to PBC in the Japanese population. The connection between genetic variants and function of the CTLA4 gene remains to be addressed in future investigations.

Key words: Primary biliary cirrhosis; Single nucleotide polymorphisms; Cytotoxic T-lymphocyte antigen 4; Genetic susceptibility 


\section{Introduction}

Primary biliary cirrhosis (PBC) is a liver-specific autoimmune disease characterized by female preponderance and destruction of intrahepatic bile ducts that often results in cirrhosis and hepatic failure [1]. The etiology of PBC has yet to be conclusively elucidated, although genetic factors are considered to play a prominent role in family and population studies [2-5]. Prior reports have shown the HLA-DRB1 08 allele to be a weak and regional determinant of PBC susceptibility [6-8]. However, HLA alone does not explain the entire genetic predisposition to PBC, mainly since at least 80 to $90 \%$ of patients with the disease do not carry the most common HLA susceptibility alleles. In this regard, other non-HLA genes are being considered to contribute to disease development $[9,10]$.

PBC displays immunologically characteristic features like biliary lymphocytic infiltrates, anti-mitochondrial antibodies (AMA) against the inner lipoyl domain of the E2 subunits of the pyruvate dehydrogenase complex, and elevated serum levels of IFN- $\gamma$ and TNF- $\alpha$. The serologic hallmark of PBC is the presence of AMA [11, 12], which are found in $95 \%$ of patients with PBC [13] and have a specificity of $98 \%$ for the disease [12]. Auto-reactive $\mathrm{CD} 4^{+}$and $\mathrm{CD} 8^{+} \mathrm{T}$ cells are also found in high concentrations in the portal triads of patients with PBC, often surrounding and infiltrating necrotic bile ducts [14-16]. A 
recent study suggested that a reduction in the number of $C D 4^{+} \mathrm{CD} 25^{+}$regulatory $\mathrm{T}$ cells in livers affected with PBC contributed to disease progression [17]. Accumulating data such as these support a direct role of T lymphocytes in the pathogenesis of PBC.

The cytotoxic T-lymphocyte Antigen 4 (CTLA4) is an inhibitory receptor expressed on the cell surface of activated memory $T$ cells and $C D 4^{+} \mathrm{CD} 25^{+}$regulatory $\mathrm{T}$ cells that acts largely as a negative regulator of T-cell responses. Since the potential inhibitory functions of CTLA4 [18] may also trigger a break-down of immunological self-tolerance, polymorphisms affecting these processes could have significant effects on susceptibility to autoimmunity.

The CTLA4 gene is a primary candidate for genetic susceptibility to autoimmune diseases, including type 1 diabetes, autoimmune hepatitis [19, 20], and autoimmune pancreatitis [21]. In particular, two single nucleotide polymorphisms (SNPs), rs231775 (49AG) and rs3087243 (CT60), have been widely studied in PBC [22-24]. Although early studies found an association between SNP 49G coding and PBC [22-24], ensuing reports showed negative relationships with susceptibility [25-30] or a positive association with liver damage [31]. A recent investigation reported that rs231725 in the 3' flanking region of CTLA4 is associated with AMA-positive PBC in Caucasians [27]. In addition to CTLA4 polymorphisms, HLA class II, IL12A, IL12RB, and several other 
candidate SNPs were disclosed as predisposition genes for PBC by a high-density genome-wide association study [9]. Since these SNPs have not been extensively examined in a large Japanese population, the present study sought to evaluate the involvement of CTLA4 SNPs and haplotype SNPs in susceptibility to PBC and disease progression in Japanese patients.

\title{
2. Patients and Methods
}

\author{
2.1. Subjects
}

We analyzed a total of 576 subjects (308 PBC patients and 268 healthy controls) collected from different two regions of Japan (Table 1). Cohort 1 consisted of 198 patients clinically diagnosed with PBC (173 women, median age 58 years old) and 170 healthy subjects who were seen at Shinshu University Hospital, Matsumoto, Japan. Cohort 2 consisted of 110 patients clinically diagnosed with PBC (92 women, median age 61 years old) and 98 healthy subjects from the National Hospital Organization Nagasaki Medical Center, Omura, Japan. The racial backgrounds of all subjects were Japanese. Control subjects were volunteers from hospital staff who had indicated the absence of any major illnesses in a standard questionnaire. The diagnosis of PBC was based on criteria from the American Association for the Study of Liver Diseases [32]. Serum AMA, 
specific for the pyruvate dehydrogenase complex-E2 component, was measured by the enzyme-linked immunosorbent assay as reported previously [33]. An index of greater than seven was considered a positive result. All patients were negative for hepatitis B surface antigen, antibody to hepatitis $\mathrm{C}$ virus, and antibody to human immunodeficiency virus. To evaluate associations between SNPs and disease progression, patients were classified into two stages based on their most recent follow-up [34]: early stage patients were histologically in Scheuer stage I or II $[35,36]$ or of unknown histological stage without liver cirrhosis, and late stage patients were histologically in Scheuer stage III or IV or clinically diagnosed with liver cirrhosis or hepatic failure. All participants provided informed written consent for this study, which was been approved by the institutional ethics committee.

\subsection{CTLA4 SNP Genotyping}

Genomic DNA from patients and controls was isolated by phenolic extraction of sodium dodecyl sulfate-lyzed and proteinase K-treated cells, as described previously [37, 38], and adjusted to $10-15 \mathrm{ng} / \mu \mathrm{l}$.

The five CTLA4 gene SNPs examined in this study (rs733618, rs5742909, rs231775, rs3087243, and rs231725) were genotyped using the 5' nuclease (TaqMan) 
assay using primer, probes, and reaction conditions as recommended by the manufacturer (Applied Biosystems, Tokyo, Japan). These SNPs were selected based on previous reports $[21-23,26,27]$, and were all located in the CTLA4 gene; SNPs rs733618 and rs5742909 were in the promoter region, SNP rs231775 in exon 1, and SNPs rs3087243 and rs231725 in the 3' untranslated region (UTR). Polymerase chain reaction was performed with a TaqMan Assay for Real-Time PCR (7500 Real Time PCR System; Applied Biosystems) following the manufacturer's instructions.

\subsection{Haplotype-Genotype Estimation}

The R package "haploview" [39] was used to evaluate the haplotype structure of the five examined CTLA4 SNPs. Pairwise linkage disequilibrium (LD) patterns and haplotype frequency analysis for all SNPs in patients and controls were assessed by the block definition by Gabriel et al. [40].

\subsection{Statistical Analysis}

The Hardy-Weinberg Equilibrium (HWE) test was done for each SNP between control and patient groups. The significance of allele distribution between PBC patients and healthy controls was assessed using the $\chi^{2}$ test with the use $2 \times 2$ or $2 \times 3$ 
comparisons. Fisher's exact probability test was used for groups with fewer than 5 samples. A $P$ value of less than 0.05 was considered statistically significant. $P$ values were corrected using Bonferroni's correction by multiplying by the number of different alleles observed in each locus $(P C)$.

\section{Results}

In total, five SNPs located in the CTLA4 gene were genotyped in 198 patients with PBC and 170 healthy controls in cohort 1 and 110 patients with PBC and 98 healthy controls in cohort 2 (Table 2). Hardy-Weinberg equilibrium (HWE) was observed for all 5 of the examined SNPs in both control groups, and the minor allele frequencies of all SNPs were greater than $5 \%$. In cohort 1 , one SNP (rs733618) differed significantly from HWE $(P=0.03)$ (Table 2), and the frequency of the minor A allele at rs231775 was significantly decreased $(33.9 \%$ vs. $41.5 \%$, odds ratio (OR) $0.72,95 \%$ confidence interval (95\% Cl) $0.53-0.99, P=0.042, P C=0.209)$ in 171 AMA-positive PBC patients compared with controls. Positivity for the major $G$ allele $(A / G+G / G)$ at $r s 231775$ was significantly higher in patients with AMA-positive PBC than in healthy subjects $(88.3 \%$ vs. $79.1 \%$, OR $1.96,95 \% \mathrm{Cl} 1.08-3.53, P=0.026, P C=0.128)$. Additionally, the allele frequency $(61.7 \%$ vs. $53.2 \%$, OR $1.41,95 \% \mathrm{Cl} 1.04-1.92, P=0.025, P C=0.127)$ and allele carrier 
frequency $(86.0 \%$ vs. $75.9 \%$, OR $1.96,95 \% \mathrm{Cl} 1.12-3.41, P=0.018, P C=0.089)$ of the major A allele at rs231725 were significantly increased in AMA-positive PBC patients compared with healthy controls. However, these statistical significances disappeared after correction for multiple testing. No significant differences were observed among the 5 SNPs in cohort 2. The allele frequency $(60.3 \%$ vs. $53.4 \%$, OR $1.33,95 \%$ Cl 1.04-1.69, $\underline{P=0.022) \text { of the major A allele at rs231725 was significantly increased in combined }}$ analysis (cohorts 1 and 2) of 273 AMA-positive PBC patients compared with 268 healthy controls (Table 3), but statistical significance was lost after correction for multiple testing $\underline{(P C=0.110)}($ Table 3). Pairwise LD mapping confirmed that all alleles were in strong LD with an index of $>0.8$. A strong LD was detected in the same block for PBC patients and controls. We next evaluated haplotype association among AMA-positive PBC patients and healthy subjects in a combined analysis. To estimate haplotype frequencies and analyze haplotype association with PBC, we selected tag SNPs using the Tagger algorithm from the Haploview program. Four tag SNPs (SNPs 2 to 5: rs5742909, rs231775, rs3087243, and rs231725) were selected to capture most of the allelic diversity in the two cohorts. The four estimated haplotypes showed a frequency of $>5 \%$ in 11 haplotypes created by expectation-maximization algorithms (Table 4). Haplotype 1 (CGGA) was significantly 
associated with AMA-positive PBC susceptibility (59.7\% vs. $51.9 \%$, OR $1.37,95 \% \mathrm{Cl}$

1.08-1.75, $P=0.0095)$. No other haplotypes were associated with either susceptibility or

resistance to $\mathrm{PBC}$.

Evaluation of the 5 CTLA4 SNPs between AMA-positive and AMA-negative

subgroups revealed neither significant allelic associations (Table 5) nor significant

haplotype associations (Table 6), even when compared for early or late stages (Table 5

and 6). Moreover, a comparison of 17 orthotopic liver transplantation (OLT) PBC cases

and 291 non-OLT cases revealed no significant differences in allele frequencies (Table 5).

$\underline{\text { In haplotype analysis, no statistical associations were found with OLT (Table 6). }}$

\section{Discussion}

This study revealed that haplotype 1 (CGGA) was significantly associated with

disease susceptibility in 273 AMA-positive PBC patients, as well as overall in all 308

PBC patients $(P=0.012)$ (data not shown). This finding is in agreement with the

Caucasian study by Juran et al. [27], and thus constitutes a promising susceptibility gene

candidate. However, since the precise function of CTLA4 SNPs remains undefined, we cannot exclude the possibility that these SNPs may only be a linkage marker for a yet unidentified SNP within the CTLA4 gene. Sequencing of the entire gene and assessing 
the functional role of these SNPs will be required.

SNP rs231775 associated with PBC is commonly referred to as $49 A G$ in several

studies [23, 24, 27, 31, 41]. Our finding corroborated a previous report [31], in which

49AG was not associated with susceptibility to PBC but there was a discrepancy in

association with liver damage that might have arisen from the number of cases analyzed.

49AG also appears to affect cell surface expression of CTLA4 by CTLA4-driven down-regulation in response to T-cell activation [42]. This coding polymorphism is located in a signal peptide that is cleaved from the functional protein, and has been shown to affect glycosylation of the autoimmune susceptibility $G$ allele, resulting in diminished processing efficiency and thus decreased trafficking to the cell surface [43]. It will be necessary to confirm the functional difference between patients with these SNPs and T-cell activation in a future study.

The rs3087243 SNP, also referred to as CT60, is located in the 3' UTR of the CTLA4 gene and reported to influence the production of the soluble isoform of CTLA4 (sCTLA4). The sCTLA4 mRNA encoded by the +CT60G-allele is produced at a reduced rate compared with that encoded by the A allele. As SCTLA4, which is secreted by resting $T$ cells, is a suppressor of T-cell activation, it is conceivable that carriers of the +CT60G-allele allele may be more susceptible to autoimmune diseases.[44] Although 
studies from Canada and Italy found an association between PBC and the CT60 SNP $[29,41]$, other studies have since failed to confirm this association $[27,28]$, including ours.

In haplotype analysis, haplotype 1 contained all of the known SNP risk alleles that have been functionally determined in other disease studies. These include the $\mathrm{C}$ allele at -318 , which has been found to affect the expression of CTLA4 mRNA cell surface expression [45], the minor $G$ allele at 49AG, reported to reduce cell surface expression of CTLA4 [42], and the G allele of CT60, which affects the expression of the soluble form of the CTLA4 molecule, indicating the possibility that this haplotype might contribute to PBC susceptibility in the Japanese population.

Lastly, Juran et al. have suggested that CTLA4 plays a role in influencing AMA development as well as progression to OLT in PBC based on their haplotype analyses [27]. Our data revealed no statistical significance in regards to AMA development or disease progression to cirrhosis or OLT, possibly due to the number of patients showing AMA negativity and proceeding to OLT being too small to evaluate. Another consideration is that disease progression in Japanese patients might have a stronger association with positivity for anti-gp210 antibodies as a risk factor of progression to hepatic failure than CTLA4 polymorphisms [46]. Further longitudinal follow-up studies in 
larger cohorts are required to resolve this critical question.

In conclusion, we found that CTLA4 gene polymorphisms had a modest, but significant, association with susceptibility to PBC in the Japanese population and may share a common susceptibility haplotype with Caucasians. The connection between genetic variants and the function of the CTLA4 gene remains to be addressed in future investigations. 


\section{Acknowledgements}

The authors who have taken part in this study declare that they have nothing to disclose

regarding funding from industries or conflicts of interest with respect to this manuscript.

We thank Yuki Akahane, Asami Yamazaki, and Toyo Amaki for their technical assistance, and Trevor Ralph for his English editorial assistance. This study was supported in part by a research grant from the Japanese Ministry of Health, Labour and Welfare and the Foundation of Nagano Prefecture for Promoting Science. 


\section{References}

[1] Talwalkar JA and Lindor KD. Primary biliary cirrhosis. Lancet 2003;362:53-61.

[2] Jones DE. Pathogenesis of primary biliary cirrhosis. J Hepatol 2003;39:639-648.

[3] Gershwin ME, Selmi C, Worman HJ, Gold EB, Watnik M, Utts J, et al. Risk factors and comorbidities in primary biliary cirrhosis: a controlled interview-based study of 1032 patients. Hepatology 2005;42:1194-1202.

[4] Kaplan MM and Gershwin ME. Primary biliary cirrhosis. N Engl J Med 2005;353:1261-1273.

[5] Gershwin ME and Mackay IR. The causes of primary biliary cirrhosis: Convenient and inconvenient truths. Hepatology 2008;47:737-745.

[6] Invernizzi P, Selmi C, Mackay IR, Podda M, and Gershwin ME. From bases to basis: linking genetics to causation in primary biliary cirrhosis. Clin Gastroenterol Hepatol 2005;3:401-410.

[7] Invernizzi P, Selmi C, Poli F, Frison S, Floreani A, Alvaro D, et al. Human leukocyte antigen polymorphisms in Italian primary biliary cirrhosis: a multicenter study of 664 patients and 1992 healthy controls. Hepatology 2008;48:1906-1912.

[8] Donaldson PT, Baragiotta A, Heneghan MA, Floreani A, Venturi C, Underhill JA, et al. HLA class II alleles, genotypes, haplotypes, and amino acids in primary biliary cirrhosis: a large-scale study. Hepatology 2006;44:667-674.

[9] Hirschfield GM, Liu X, Xu C, Lu Y, Xie G, Gu X, et al. Primary biliary cirrhosis associated with HLA, IL12A, and IL12RB2 variants. N Engl J Med 2009;360:2544-2555.

[10] Joshita S, Umemura T, Yoshizawa K, Katsuyama Y, Tanaka E, and Ota M. A2BP1 as a novel susceptible gene for primary biliary cirrhosis in Japanese patients. Hum Immunol 2010;Mar 6. [Epub ahead of print] in press.

[11] Shimoda S, Nakamura M, Ishibashi H, Hayashida K, and Niho Y. HLA DRB4 0101-restricted immunodominant T cell autoepitope of pyruvate dehydrogenase complex in primary biliary cirrhosis: 
evidence of molecular mimicry in human autoimmune diseases. J Exp Med 1995;181:1835-1845.

[12] Van de Water J, Cooper A, Surh CD, Coppel R, Danner D, Ansari A, et al. Detection of autoantibodies to recombinant mitochondrial proteins in patients with primary biliary cirrhosis. $\mathrm{N}$ Engl J Med 1989;320:1377-1380.

[13] Miyakawa H, Tanaka A, Kikuchi K, Matsushita M, Kitazawa E, Kawaguchi N, et al. Detection of antimitochondrial autoantibodies in immunofluorescent AMA-negative patients with primary biliary cirrhosis using recombinant autoantigens. Hepatology 2001;34:243-248.

[14] Colucci G, Schaffner F, and Paronetto F. In situ characterization of the cell-surface antigens of the mononuclear cell infiltrate and bile duct epithelium in primary biliary cirrhosis. Clin Immunol Immunopathol 1986;41:35-42.

[15] Shimoda S, Van de Water J, Ansari A, Nakamura M, Ishibashi H, Coppel RL, et al. Identification and precursor frequency analysis of a common $\mathrm{T}$ cell epitope motif in mitochondrial autoantigens in primary biliary cirrhosis. J Clin Invest 1998;102:1831-1840.

[16] Migliaccio C, Van de Water J, Ansari AA, Kaplan MM, Coppel RL, Lam KS, et al. Heterogeneous response of antimitochondrial autoantibodies and bile duct apical staining monoclonal antibodies to pyruvate dehydrogenase complex E2: the molecule versus the mimic. Hepatology 2001;33:792-801.

[17] Lan RY, Cheng C, Lian ZX, Tsuneyama K, Yang GX, Moritoki Y, et al. Liver-targeted and peripheral blood alterations of regulatory T cells in primary biliary cirrhosis. Hepatology 2006;43:729-737.

[18] Gough SC, Walker LS, and Sansom DM. CTLA4 gene polymorphism and autoimmunity. Immunol Rev 2005;204:102-115.

[19] Agarwal K, Czaja AJ, Jones DE, and Donaldson PT. Cytotoxic T lymphocyte antigen-4 (CTLA-4) gene polymorphisms and susceptibility to type 1 autoimmune hepatitis. Hepatology 2000;31:49-53.

[20] Umemura T, Ota M, Yoshizawa K, Katsuyama Y, Ichijo T, Tanaka E, et al. Association of cytotoxic T-lymphocyte antigen 4 gene polymorphisms with type 1 autoimmune hepatitis in Japanese. Hepatol 
Res 2008;38:689-695.

[21] Umemura $\mathrm{T}$, Ota $\mathrm{M}$, Hamano $\mathrm{H}$, Katsuyama $\mathrm{Y}$, Muraki $\mathrm{T}$, Arakura $\mathrm{N}$, et al. Association of autoimmune pancreatitis with cytotoxic T-lymphocyte antigen 4 gene polymorphisms in Japanese patients. Am J Gastroenterol 2008;103:588-594.

[22] Agarwal K, Jones DE, Daly AK, James OF, Vaidya B, Pearce S, et al. CTLA-4 gene polymorphism confers susceptibility to primary biliary cirrhosis. J Hepatol 2000;32:538-541.

[23] Fan LY, Tu XQ, Cheng QB, Zhu Y, Feltens R, Pfeiffer T, et al. Cytotoxic T lymphocyte associated antigen-4 gene polymorphisms confer susceptibility to primary biliary cirrhosis and autoimmune hepatitis in Chinese population. World J Gastroenterol 2004;10:3056-3059.

[24] Poupon R, Ping C, Chretien Y, Corpechot C, Chazouilleres O, Simon T, et al. Genetic factors of susceptibility and of severity in primary biliary cirrhosis. J Hepatol 2008;49:1038-1045.

[25] Bittencourt PL, Palacios SA, Farias AQ, Abrantes-Lemos CP, Cancado EL, Carrilho FJ, et al. Analysis of major histocompatibility complex and CTLA-4 alleles in Brazilian patients with primary biliary cirrhosis. J Gastroenterol Hepatol 2003;18:1061-1066.

[26] Donaldson P, Veeramani S, Baragiotta A, Floreani A, Venturi C, Pearce S, et al. Cytotoxic T-lymphocyte-associated antigen-4 single nucleotide polymorphisms and haplotypes in primary biliary cirrhosis. Clin Gastroenterol Hepatol 2007;5:755-760.

[27] Juran BD, Atkinson EJ, Schlicht EM, Fridley BL, and Lazaridis KN. Primary biliary cirrhosis is associated with a genetic variant in the $3^{\prime}$ flanking region of the CTLA4 gene. Gastroenterology 2008;135:1200-1206.

[28] Juran BD, Atkinson EJ, Schlicht EM, Fridley BL, Petersen GM, and Lazaridis KN. Interacting alleles of the coinhibitory immunoreceptor genes cytotoxic T-lymphocyte antigen 4 and programmed cell-death 1 influence risk and features of primary biliary cirrhosis. Hepatology 2008;47:563-570.

[29] Oertelt S, Kenny TP, Selmi C, Invernizzi P, Podda M, and Gershwin ME. SNP analysis of genes 
implicated in T cell proliferation in primary biliary cirrhosis. Clin Dev Immunol 2005;12:259-263.

[30] Schott E, Witt H, Pascu M, van Boemmel F, Weich V, Bergk A, et al. Association of CTLA4 single nucleotide polymorphisms with viral but not autoimmune liver disease. Eur J Gastroenterol Hepatol 2007;19:947-951.

[31] Kanno Y, Rai T, Monoe K, Saito H, Takahashi A, Irisawa A, et al. Possible association of cytotoxic T lymphocyte antigen-4 genetic polymorphism with liver damage of primary biliary cirrhosis in Japan. Fukushima J Med Sci 2006;52:79-85.

[32] Lindor KD, Gershwin ME, Poupon R, Kaplan M, Bergasa NV, and Heathcote EJ. Primary biliary cirrhosis. Hepatology 2009;50:291-308.

[33] Umemura $\mathrm{T}$, Zen $\mathrm{Y}$, Hamano $\mathrm{H}$, Kawa $\mathrm{S}$, Nakanuma $\mathrm{Y}$, and Kiyosawa $\mathrm{K}$. Immunoglobin G4-hepatopathy: association of immunoglobin G4-bearing plasma cells in liver with autoimmune pancreatitis. Hepatology 2007;46:463-471.

[34] Nakamura M, Yasunami M, Kondo H, Horie H, Aiba Y, Komori A, et al. Analysis of HLA-DRB1 polymorphisms in Japanese patients with primary biliary cirrhosis (PBC): the HLA-DRB1 polymorphism determines the relative risk of antinuclear antibodies for disease progression in PBC. Hepatol Res 2010;in press.

[35] Scheuer PJ. Ludwig Symposium on biliary disorders--part II. Pathologic features and evolution of primary biliary cirrhosis and primary sclerosing cholangitis. Mayo Clin Proc 1998;73:179-183.

[36] Ludwig J, Dickson ER, and McDonald GS. Staging of chronic nonsuppurative destructive cholangitis (syndrome of primary biliary cirrhosis). Virchows Arch A Pathol Anat Histol 1978;379:103-112.

[37] Ota M, Seki T, Fukushima H, Tsuji K, and Inoko H. HLA-DRB1 genotyping by modified PCR-RFLP method combined with group-specific primers. Tissue Antigens 1992;39:187-202.

[38] Ota M, Seki T, Nomura N, Sugimura K, Mizuki N, Fukushima H, et al. Modified PCR-RFLP method for HLA-DPB1 and -DQA1 genotyping. Tissue Antigens 1991;38:60-71. 
[39] Barrett JC, Fry B, Maller J, and Daly MJ. Haploview: analysis and visualization of LD and haplotype maps. Bioinformatics 2005;21:263-265.

[40] Gabriel SB, Schaffner SF, Nguyen H, Moore JM, Roy J, Blumenstiel B, et al. The structure of haplotype blocks in the human genome. Science 2002;296:2225-2229.

[41] Walker EJ, Hirschfield GM, Xu C, Lu Y, Liu X, Coltescu C, et al. CTLA4/ICOS gene variants and haplotypes are associated with rheumatoid arthritis and primary biliary cirrhosis in the Canadian population. Arthritis Rheum 2009;60:931-937.

[42] Maurer M, Loserth S, Kolb-Maurer A, Ponath A, Wiese S, Kruse N, et al. A polymorphism in the human cytotoxic T-lymphocyte antigen 4 ( CTLA4) gene (exon $1+49)$ alters T-cell activation. Immunogenetics 2002;54:1-8.

[43] Anjos S, Nguyen A, Ounissi-Benkalha H, Tessier MC, and Polychronakos C. A common autoimmunity predisposing signal peptide variant of the cytotoxic T-lymphocyte antigen 4 results in inefficient glycosylation of the susceptibility allele. J Biol Chem 2002;277:46478-46486.

[44] Ueda H, Howson JM, Esposito L, Heward J, Snook H, Chamberlain G, et al. Association of the T-cell regulatory gene CTLA4 with susceptibility to autoimmune disease. Nature 2003;423:506-511.

[45] Ligers A, Teleshova N, Masterman T, Huang WX, and Hillert J. CTLA-4 gene expression is influenced by promoter and exon 1 polymorphisms. Genes Immun 2001;2:145-152.

[46] Nakamura $M$, Kondo $H$, Mori $T$, Komori A, Matsuyama $M$, Ito $M$, et al. Anti-gp210 and anti-centromere antibodies are different risk factors for the progression of primary biliary cirrhosis. Hepatology 2007;45:118-127. 
Table 1. Demographic and clinical data of patients with PBC at study onset

\begin{tabular}{|c|c|c|c|}
\hline$\underline{\text { Characteristics }}$ & $\begin{array}{l}\frac{\text { Cohort } 1}{\text { Shinshu }} \\
\underline{\mathrm{n}=198}\end{array}$ & $\begin{array}{c}\frac{\text { Cohort 2 }}{\text { Nagasaki }} \\
\underline{\mathrm{n}=110}\end{array}$ & Combined \\
\hline Age, yearst & $\underline{58(30-83)}$ & $\underline{61(34-85)}$ & $\underline{58(30-88)}$ \\
\hline Female / Male & $173 / 25$ & $\underline{92 / 18}$ & $265 / 43$ \\
\hline \multicolumn{4}{|l|}{ Disease progression } \\
\hline Early stage, $\mathrm{n} /$ Late stage, $\mathrm{n}$ & $\underline{149 / 49}$ & $\underline{74 / 36}$ & $\underline{223 / 85}$ \\
\hline Orthotopic liver transplantation, n (\%) & $\underline{15(7.6)}$ & $\underline{2(1.8)}$ & $\underline{17(5.5)}$ \\
\hline AMA positive, $\mathrm{n}(\%)$ & $\underline{171(86.4)}$ & $\underline{102(92.8)}$ & $273(88.6)$ \\
\hline
\end{tabular}

t Median (range)

PBC, primary biliary cirrhosis; AMA, anti-mitochondrial antibody specific for the pyruvate dehydrogenase complex-E2 component 
Table 2. Allele frequencies of SNPs in the CTLA4 gene in PBC patients and controls

\begin{tabular}{|c|c|c|c|c|c|c|c|c|c|c|c|c|}
\hline \multirow{2}{*}{ SNP No. } & \multirow{2}{*}{$\mathrm{dbSNP}$} & \multirow{2}{*}{$\begin{array}{l}\text { Allele } \\
\text { ajor/minor }\end{array}$} & \multirow{2}{*}{$\begin{array}{l}\text { Position } \\
\text { (bp) }\end{array}$} & \multirow{2}{*}{$\begin{array}{c}\text { Gene } \\
\text { location }\end{array}$} & \multicolumn{4}{|c|}{ Cohort 1 (Shinshu) } & \multicolumn{4}{|c|}{ Cohort 2 (Nagasaki) } \\
\hline & & & & & $\underline{M A F}$ & HWE & $\underline{M A F}$ & HWE & MAF & HWE & MAF & $\underline{H W E}$ \\
\hline 1 & rs733618 & $\mathrm{T} / \mathrm{C}$ & 204439189 & promoter & $\underline{44.4}$ & $\underline{0.030}$ & $\underline{39.1}$ & $\underline{0.071}$ & $\underline{39.5}$ & $\underline{0.570}$ & $\underline{43.4}$ & $\underline{0.366}$ \\
\hline 2 & rs5742909 & $\mathrm{C} / \mathrm{T}$ & 204440592 & promoter & $\underline{9.1}$ & $\underline{0.347}$ & $\underline{11.2}$ & $\underline{0.295}$ & $\underline{13.2}$ & $\underline{0.828}$ & $\underline{13.8}$ & $\underline{0.514}$ \\
\hline 4 & rs3087243 & $\mathrm{G} / \mathrm{A}$ & 204447164 & 3' UTR & $\underline{26.3}$ & $\underline{0.994}$ & $\underline{30.3}$ & $\underline{0.709}$ & $\underline{26.4}$ & $\underline{0.125}$ & $\underline{31.1}$ & $\underline{0.316}$ \\
\hline 5 & rs231725 & $A / G$ & 204448920 & 3' UTR & $\underline{39.9}$ & $\underline{1.000}$ & $\underline{46.8}$ & $\underline{0.288}$ & $\underline{41.8}$ & $\underline{0.586}$ & $\underline{46.4}$ & $\underline{1.000}$ \\
\hline
\end{tabular}

MAF, minor allele frequency; HWE, Hardy-Weinberg equilibrium; UTR, untranslated region 
Table 3.

1

2

3

4

5

6

7

8

9

10

11

12

13

14

15

16

17

18

19

20

21

22

23

24

25

26

27

28

Allele frequencies of 5 SNPs in $273 \mathrm{AMA}^{+}$patients with PBC and 268 healthy subjects

\begin{tabular}{|c|c|c|c|c|c|c|c|}
\hline SNP No. & Allele & Patients & Controls & $\underline{P}$ & $\underline{P C}$ & $\underline{O R}$ & $95 \% \mathrm{Cl}$ \\
\hline \multirow[t]{2}{*}{1} & $\underline{\mathrm{C}}$ & 43.2 & $\underline{40.7}$ & $\underline{0.395}$ & 1.975 & $\underline{1.11}$ & $\underline{0.87-1.41}$ \\
\hline & $\underline{T}$ & $\underline{56.8}$ & $\underline{59.3}$ & & & & \\
\hline \multirow[t]{2}{*}{$\underline{2}$} & $\underline{\mathrm{C}}$ & $\underline{89.6}$ & $\underline{87.9}$ & $\underline{0.380}$ & 1.900 & $\underline{1.18}$ & $\underline{0.81-1.73}$ \\
\hline & I & $\underline{10.4}$ & $\underline{12.1}$ & & & & \\
\hline \multirow[t]{2}{*}{$\underline{3}$} & $\underline{\mathrm{G}}$ & $\underline{63.9}$ & $\underline{58.4}$ & $\underline{0.062}$ & $\underline{0.310}$ & $\underline{1.26}$ & $\underline{0.99-1.61}$ \\
\hline & $\underline{A}$ & $\underline{36.1}$ & $\underline{41.6}$ & & & & \\
\hline \multirow[t]{2}{*}{$\underline{4}$} & $\underline{G}$ & $\underline{74.4}$ & $\underline{69.4}$ & $\underline{0.070}$ & $\underline{0.350}$ & $\underline{1.28}$ & $\underline{0.98-1.67}$ \\
\hline & $\underline{A}$ & $\underline{25.6}$ & $\underline{30.6}$ & & & & \\
\hline \multirow[t]{2}{*}{$\underline{5}$} & $\underline{A}$ & $\underline{60.3}$ & $\underline{53.4}$ & $\underline{0.022}$ & $\underline{0.110}$ & $\underline{1.33}$ & $\underline{1.04-1.69}$ \\
\hline & $\underline{\mathrm{G}}$ & 39.7 & $\underline{56.6}$ & & & & \\
\hline
\end{tabular}

AMA, anti-mitochondrial antibodies; PBC, primary biliary cirrhosis; OR, odds ratio; $P c$, corrected $P$ value; $95 \%$ $\mathrm{Cl}, 95 \%$ confidence interval; ; , frequency (\%)

$P$ value was calculated by a $\chi^{2}$-test $2 \times 2$ contingency table $(\mathrm{df}=1)$. 
Table 4. CTLA4 haplotypes in $273 \mathrm{AMA}^{+}$patients with PBC and 268 healthy subjects

\begin{tabular}{|c|c|c|c|c|c|c|c|c|c|}
\hline \multirow{2}{*}{$\underline{\text { Haplotype }}$} & \multicolumn{4}{|c|}{ SNP No. } & \multirow{2}{*}{$\frac{\text { Patients }^{*}}{(n=546)}$} & \multirow{2}{*}{$\begin{array}{c}\text { Controls }^{*} \\
(n=536)\end{array}$} & \multirow[b]{2}{*}{$\underline{P}$} & \multirow[b]{2}{*}{$\underline{\mathrm{OR}}$} & \multirow[b]{2}{*}{$95 \% \mathrm{Cl}$} \\
\hline & $\underline{2}$ & $\underline{3}$ & $\underline{4}$ & $\underline{5}$ & & & & & \\
\hline 1 & $\underline{\mathrm{C}}$ & $\underline{\mathrm{G}}$ & $\underline{\mathrm{G}}$ & $\underline{A}$ & $\underline{59.7}$ & $\underline{51.9}$ & $\underline{0.0095}$ & $\underline{1.37}$ & $\underline{1.08-1.75}$ \\
\hline$\underline{2}$ & $\underline{\mathrm{C}}$ & $\underline{A}$ & $\underline{A}$ & $\underline{\mathrm{G}}$ & $\underline{25.5}$ & $\underline{29.4}$ & $\underline{0.1464}$ & $\underline{0.82}$ & $\underline{0.62-1.07}$ \\
\hline$\underline{3}$ & $\underline{T}$ & $\underline{A}$ & $\underline{\mathrm{G}}$ & $\underline{\mathrm{G}}$ & $\underline{10.3}$ & $\underline{11.8}$ & $\underline{0.4186}$ & $\underline{0.85}$ & $\underline{0.58-1.25}$ \\
\hline$\underline{4}$ & $\underline{\mathrm{C}}$ & $\underline{\mathrm{G}}$ & $\underline{\mathrm{G}}$ & $\underline{\mathrm{G}}$ & $\underline{3.8}$ & $\underline{5.4}$ & $\underline{0.2153}$ & $\underline{0.70}$ & $\underline{0.39-1.23}$ \\
\hline
\end{tabular}

PBC, primary biliary cirrhosis; OR, odds ratio; $95 \% \mathrm{Cl}, 95 \%$ confidence interval; * , Proportion of indicated haplotype (\%)

Values for $\mathrm{n}$ indicate two times the number of individuals since each person carries two haplotypes.

$P$ value was calculated by a $\chi^{2}$-test $2 \times 2$ contingency table $(\mathrm{df}=1)$. 
Table 5. Allele frequencies of CTLA4 SNPs in AMA, histological or clinical disease progression, and OLT states

\begin{tabular}{|c|c|c|c|c|c|c|c|c|c|c|}
\hline \multirow[b]{2}{*}{ SNP No. } & \multirow[b]{2}{*}{ Allele } & $\underline{\mathrm{AMA}^{+*}}$ & \multicolumn{2}{|l|}{$\underline{\mathrm{AMA}^{-*}}$} & \multirow{2}{*}{$\begin{array}{l}\text { Early * } \\
(\mathrm{n}=223) \\
\end{array}$} & \multicolumn{2}{|l|}{$\underline{\text { Late }^{*}}$} & \multirow{2}{*}{$\frac{\text { non-OLT * }}{\underline{(n=291)}}$} & \multicolumn{2}{|l|}{$\underline{\mathrm{OLT}^{*}}$} \\
\hline & & $(n=273)$ & $(\mathrm{n}=35)$ & $\underline{P}$ & & $(n=85)$ & $\underline{P}$ & & $(n=17)$ & $\underline{P}$ \\
\hline \multirow[t]{2}{*}{1} & $\underline{\mathrm{C}}$ & $\underline{43.2}$ & $\underline{38.6}$ & $\underline{0.459}$ & $\underline{44.4}$ & $\underline{38.2}$ & $\underline{0.167}$ & $\underline{42.6}$ & $\underline{44.1}$ & $\underline{0.863}$ \\
\hline & I & $\underline{56.8}$ & $\underline{61.4}$ & & $\underline{55.6}$ & $\underline{61.8}$ & & $\underline{57.4}$ & $\underline{55.9}$ & \\
\hline \multirow[t]{2}{*}{$\underline{2}$} & $\underline{C}$ & $\underline{89.6}$ & $\underline{88.6}$ & $\underline{0.800}$ & $\underline{90.0}$ & $\underline{89.2}$ & $\underline{0.783}$ & $\underline{89.3}$ & $\underline{91.2}$ & $\underline{0.736}$ \\
\hline & I & $\underline{10.4}$ & $\underline{11.4}$ & & $\underline{10.0}$ & $\underline{10.8}$ & & $\underline{10.7}$ & $\underline{8.8}$ & \\
\hline \multirow[t]{2}{*}{$\underline{3}$} & $\underline{G}$ & $\underline{63.9}$ & $\underline{57.1}$ & $\underline{0.267}$ & $\underline{63.9}$ & $\underline{61.2}$ & $\underline{0.531}$ & $\underline{62.9}$ & $\underline{67.6}$ & $\underline{0.576}$ \\
\hline & $\underline{A}$ & $\underline{36.1}$ & $\underline{42.9}$ & & $\underline{36.1}$ & $\underline{38.8}$ & & $\underline{37.1}$ & $\underline{32.4}$ & \\
\hline \multirow[t]{2}{*}{$\underline{4}$} & $\underline{\mathrm{G}}$ & $\underline{74.4}$ & $\underline{68.6}$ & $\underline{0.300}$ & $\underline{74.7}$ & $\underline{71.2}$ & $\underline{0.380}$ & $\underline{73.7}$ & $\underline{73.5}$ & $\underline{0.981}$ \\
\hline & $\underline{A}$ & $\underline{25.6}$ & $\underline{31.4}$ & & $\underline{25.3}$ & $\underline{28.8}$ & & $\underline{26.3}$ & $\underline{26.5}$ & \\
\hline \multirow[t]{2}{*}{$\underline{5}$} & $\underline{A}$ & $\underline{60.3}$ & $\underline{52.9}$ & $\underline{0.235}$ & $\underline{60.8}$ & $\underline{55.9}$ & $\underline{0.270}$ & $\underline{59.5}$ & $\underline{58.8}$ & $\underline{0.942}$ \\
\hline & $\underline{\mathrm{G}}$ & 39.7 & $\underline{47.1}$ & & 39.2 & $\underline{44.1}$ & & $\underline{40.5}$ & $\underline{41.2}$ & \\
\hline
\end{tabular}

PBC, primary biliary cirrhosis; AMA, anti-mitochondrial antibodies; OLT, orthotopic liver transplantation;

SNP, single nucleotide polymorphism; *, frequency (\%)

$P$ value was calculated by a $\chi^{2}$-test $2 \times 2$ contingency table $(\mathrm{df}=1)$. 
8Table 6. Comparison of CTLA4 haplotype frequencies in AMA, histological or clinical disease progression, and OLT states

\begin{tabular}{|c|c|c|c|c|c|c|c|c|c|c|c|c|c|}
\hline \multirow{2}{*}{$\begin{array}{l}10 \\
11 \text { Haplotype } \\
12\end{array}$} & \multicolumn{4}{|c|}{ SNPs No. } & \multirow{2}{*}{$\frac{\mathrm{AMA}^{+*}}{(\mathrm{n}=546)}$} & \multicolumn{2}{|l|}{$\mathrm{AMA}^{-*}$} & \multirow{2}{*}{$\begin{array}{l}\text { Early * } \\
(\mathrm{n}=446) \\
\end{array}$} & \multicolumn{2}{|l|}{ Late * } & \multirow{2}{*}{$\frac{\text { non-OLT * }}{\underline{(n=582)}}$} & \multicolumn{2}{|l|}{$\underline{\mathrm{OLT}}{ }^{*}$} \\
\hline & $\underline{2}$ & $\underline{3}$ & $\underline{4}$ & $\underline{5}$ & & $(n=70)$ & $\underline{P}$ & & $(n=170)$ & $\underline{P}$ & & $(n=34)$ & $\underline{P}$ \\
\hline $\begin{array}{l}13 \\
14\end{array}$ & $\underline{\mathrm{C}}$ & $\underline{\mathrm{G}}$ & $\underline{\mathrm{G}}$ & $\underline{A}$ & $\underline{60.1}$ & $\underline{52.8}$ & $\underline{0.245}$ & $\underline{60.5}$ & $\underline{55.9}$ & $\underline{0.292}$ & $\underline{59.3}$ & $\underline{58.8}$ & $\underline{0.959}$ \\
\hline 15 & $\underline{\mathrm{C}}$ & $\underline{A}$ & $\underline{A}$ & $\underline{\mathrm{G}}$ & $\underline{25.5}$ & $\underline{30.0}$ & $\underline{0.415}$ & 25.1 & $\underline{28.2}$ & $\underline{0.430}$ & 26.1 & $\underline{23.5}$ & $\underline{0.738}$ \\
\hline 17 & I & $\underline{A}$ & $\underline{\mathrm{G}}$ & $\underline{\mathrm{G}}$ & $\underline{10.3}$ & $\underline{10.0}$ & $\underline{0.947}$ & $\underline{10.3}$ & $\underline{10.0}$ & $\underline{0.909}$ & $\underline{10.3}$ & $\underline{8.8}$ & $\underline{0.781}$ \\
\hline $\begin{array}{l}18 \\
19\end{array}$ & $\underline{\mathrm{C}}$ & $\underline{G}$ & $\underline{\mathrm{G}}$ & $\underline{G}$ & $\underline{3.5}$ & $\underline{4.3}$ & $\underline{0.720}$ & 3.1 & $\underline{4.7}$ & $\underline{0.346}$ & 3.4 & $\underline{5.9}$ & $\underline{0.458}$ \\
\hline
\end{tabular}

21 PBC, primary biliary cirrhosis; AMA, anti-mitochondrial antibodies; OLT, orthotopic liver transplantation; SNP, single nucleotide polymorphism; ${ }^{*}$, Proportion of indicated

24haplotype (\%)

26

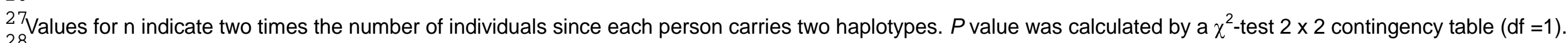

29

30

31

32

34

36

37

38

39

40

42

43

45

46 